\title{
TITLE:
}

\section{Damping filter method for obtaining spatially localized solutions}

AUTHOR(S):

Teramura, Toshiki; Toh, Sadayoshi

CITATION:

Teramura, Toshiki ...[et al]. Damping filter method for obtaining spatially localized solutions. Physical Review E 2014, 89(5): 052910.

ISSUE DATE:

2014-05-14

URL:

http://hdl.handle.net/2433/188916

RIGHT:

C 2014 American Physical Society 
PHYSICAL REVIEW E 89, 052910 (2014)

\title{
Damping filter method for obtaining spatially localized solutions
}

\author{
Toshiki Teramura* and Sadayoshi Toh \\ Department of Physics and Astronomy, Graduate School of Science, Kyoto University, Japan
}

(Received 14 November 2013; published 14 May 2014)

\begin{abstract}
Spatially localized structures are key components of turbulence and other spatiotemporally chaotic systems. From a dynamical systems viewpoint, it is desirable to obtain corresponding exact solutions, though their existence is not guaranteed. A damping filter method is introduced to obtain variously localized solutions and adapted in two typical cases. This method introduces a spatially selective damping effect to make a good guess at the exact solution, and we can obtain an exact solution through a continuation with the damping amplitude. The first target is a steady solution to the Swift-Hohenberg equation, which is a representative of bistable systems in which localized solutions coexist and a model for spanwise-localized cases. Not only solutions belonging to the well-known snaking branches but also those belonging to isolated branches known as "isolas" are found with continuation paths between them in phase space extended with the damping amplitude. This indicates that this spatially selective excitation mechanism has an advantage in searching spatially localized solutions. The second target is a spatially localized traveling-wave solution to the Kuramoto-Sivashinsky equation, which is a model for streamwise-localized cases. Since the spatially selective damping effect breaks Galilean and translational invariances, the propagation velocity cannot be determined uniquely while the damping is active, and a singularity arises when these invariances are recovered. We demonstrate that this singularity can be avoided by imposing a simple condition, and a localized traveling-wave solution is obtained with a specific propagation speed.
\end{abstract}

DOI: 10.1103/PhysRevE.89.052910

\section{INTRODUCTION}

A dynamical systems point of view and the accompanying exact solutions to the Navier-Stokes equation play key roles in understanding the dynamics of turbulence [1]. It is useful both in transient flows and statistically steady flows. Indeed, in minimal turbulence [2], a crucial aspect of the dynamics of laminar-turbulent transition processes has been revealed by the discovery of the "edge state" [3,4], and that of the self-sustaining process (SSP) has been done by the discovery of unstable periodic orbits which reproduce the statistics of turbulence [5].

The dynamical systems viewpoint, however, has not yet successfully captured the full nonlinear spatiotemporal dynamics of turbulence. One major limitation is that there is no general framework for obtaining solutions corresponding to spatially localized structures in turbulence. For example, in channel flows there exist near-wall structures and large scale motion (LSM) that occupies the outer layer above the near-wall layer. In order to elucidate their intrinsic dynamics and interactions among them from the dynamical systems viewpoint, it is desireable to obtain corresponding exact solutions separately. Such localized exact solutions have received much attention in recent years and indeed have been obtained in a few cases [6-9]. However, despite their importance, any practical ways of obtaining them have not been established yet. For this purpose, we introduce a damping filter method in Sec. II.

There exist various types of localized structures. For example, turbulent puffs in pipe flow are localized in the streamwise direction; turbulent spots in channel flow do both in the streamwise and spanwise directions. If there exists a

\footnotetext{
*teramura@kyoryu.scphys.kyoto-u.ac.jp
}

PACS number(s): 05.45.Yv, 47.27.ed, 47.10.Fg, 47.27.nd

solution corresponding to LSM, it will be localized in the wallnormal direction. In this paper, we focus on the two typical cases, both of which will be instructive in understanding localized structures observed in turbulence. We show not only basic usage and results but also remarkable features of our method in Secs. III and IV.

We first consider localized solutions to the SwiftHohenberg equation (SHE) [10-12]. This is a representative example of localized solutions in bistable systems. This class of localized solutions contains, for example, spanwiselocalized solutions corresponding to roll-streak structures in plane Couette flow [13]. Their solution branches are very similar to the "snaking" branches seen in SHE [10]. Similar structures of solution branches are also found in doubly diffusive convection systems [14]. These facts indicate that there exists a universal mechanism of spatially localized solutions in the bistable systems. We deal with solutions of this class in Sec. III.

Second, we examine a spatially localized traveling-wave solution to the Kuramoto-Sivashinsky equation. Such a solution can be regarded as a streamwise-localized solution. Streamwise-localized structures can be observed in pipe flow (turbulent puffs), boundary layers (hairpin vortices), and so on. The sustaining mechanism of them might differ from that of spanwisely-localized solutions, and thus it is necessary to obtain the corresponding solutions in order to analyze them from a dynamical systems viewpoint. At a glance, since our method utilizes a spatially selective damping effect that breaks translational invariance, it seems to have only limited capability for this issue. However, we show that this is not the case in Sec. IV.

This paper is organized as follows. In Sec. II, a damping filter method is introduced, where we explain its concept and concrete procedure. In Secs. III and IV we adapt this method to the Swift-Hohenberg equation and the Kuramoto-Sivashinsky 
(i) Introduce $\Omega$ and the filtered equation (1)

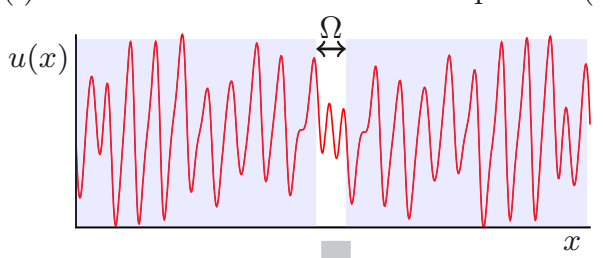

(ii) DNS yields a stable solution to Eq. (1)

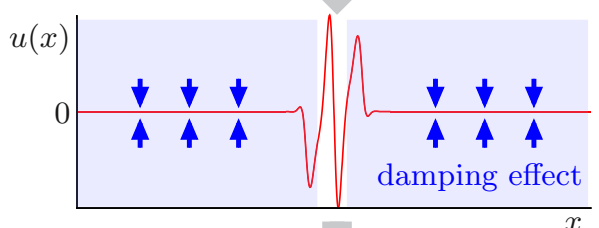

(iii) Remove the damping filter $\left(A_{f} \rightarrow 0\right)$

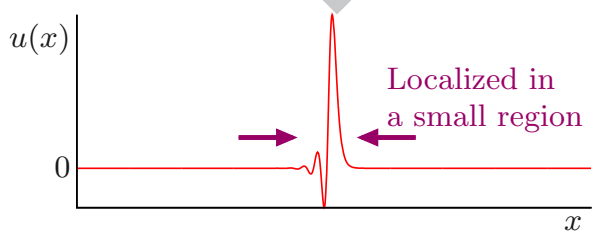

FIG. 1. (Color online) Three steps in the damping filtering method.

equation, respectively, in order to obtain spatially localized solutions. Finally, this paper is concluded in Sec. V.

\section{DAMPING FILTER METHOD}

In this section the protocol of the damping filter method is explained. This method consists of three steps (Fig. 1). Since this method can be used for various systems, a general form evolution equation, $\partial_{t} u=F[u]$, is used in the following explanation.

The first step of this method is to introduce a spatially selective damping term into the evolution equation. The damping term works only in a region $\Omega$. If we want to obtain a spanwise-localized solution, $\Omega$ should be a region localized in the spanwise direction. If we want to obtain a solution localized like turbulent spot, $\Omega$ should be a spot region. Then the damping term is introduced as follows:

$$
\partial_{t} u(x, t)=F[u](x, t)-A_{f} H(x) u(x, t) .
$$

We call this equation a "filtered equation" hereafter. The damping term consists of the filter amplitude $A_{f}$ and a filter function $H(x) . H(x)$ is defined to be zero in the region $\Omega$ and to be 1 out of $\Omega$, and smoothed by taking a convolution with a mean-zero Gaussian $\mathcal{N}_{0, \sigma^{2}}(x)$ in order to avoid the numerical singularity,

$$
\begin{gathered}
H(x)=\int \mathcal{N}_{0, \sigma^{2}}(x-y) \hat{H}(y) d y, \\
\hat{H}(x)=\left\{\begin{array}{ll}
0 & (x \in \Omega) \\
1 & (x \notin \Omega)
\end{array} .\right.
\end{gathered}
$$

The integral is taken in the whole region. The damping term causes a linear damping effect out of $\Omega$ (filtered region), and the filtered equation equals to the original equation $\partial_{t} u=F[u]$ in $\Omega$ (unfiltered region).
The second step is to obtain an exact solution to the filtered equation (1). Due to the spatially selective damping effect, the direct numerical simulation (DNS) of the filtered equation tends to yield a spatially localized time series $u(x, t)$; in other words, $u(x, t)$ decreases exponentially fast as $x$ goes away from $\Omega$ after a relaxation time. In addition, since the damping effect weakens the instability of the system, DNS sometimes yields a nontrivial stable solution for enough-large filter amplitudes and an appropriate $\Omega$. In this case the second step is finished with this stable solution. If any stable solutions are not obtained, a solution to the filtered equation is obtained by solving an equation $F[u]-A_{f} H(x) u=0$ about $u(x)$ with the Newton method. Since the degree of freedom is also reduced by the damping term, it is expected that such a solution can be obtained easily.

The third step is a continuation process. The solution obtained in the second step depends on the filter amplitude $A_{f}$, and often this dependency is continuous. A continuation with $A_{f}$ is started from this solution. The filter amplitude $A_{f}$ is decreased until it gets to zero, where the filtered equation is restored to the original equation in the whole region. Then the solution yielded from this continuation is nothing but that to the original equation. This is the goal of the damping filter method. The continuation is implemented by use of the arc-length method with the Newton-Krylov iterative method and thus is applicable to systems having a large degree of freedom.

The good feature of our method is that an appropriate guess of a spatially localized solution is constructed as a solution to the filtered equation (1). This guess reflects the dynamics of spatially localized structures since the filtered equation is equal to the original equation in the region $\Omega$. In another study [15], an artificial external forcing is used for constructing a guess of solutions. It was designed by hand from the inference about the dynamics of localized structures. In our method such artificial manipulation is not needed except for determining the region $\Omega$.

This spatially selective damping is inspired by the work in Ref. [16]. They have investigated an autonomous behaviors of near-wall structures by a filtered dynamics. In contrast to them, our method uses this filtered dynamics only for guesses and continuations and removes the filter finally. Thus, our method enable us to study the nonfiltered dynamics by localized solutions.

\section{SPANWISE-LOCALIZED SOLUTIONS}

In this section we consider the one-dimensional SHE,

$$
\frac{\partial u}{\partial t}=F[u]=\left[r-\left(\frac{\partial^{2}}{\partial x^{2}}+1\right)^{2}\right] u+2 u^{3}-u^{5} .
$$

As noted in the Introduction, a series of solutions to SHE is regarded as a representative of localized solutions in the bistable systems. The following subsections show two things: (i) We can obtain spanwise-localized solutions by use of our method. In order to show this, we reproduce solutions belonging to the homoclinic snaking branches. The practical details of our method are also described. (ii) Our method has a capability for obtaining various solutions that are usually 


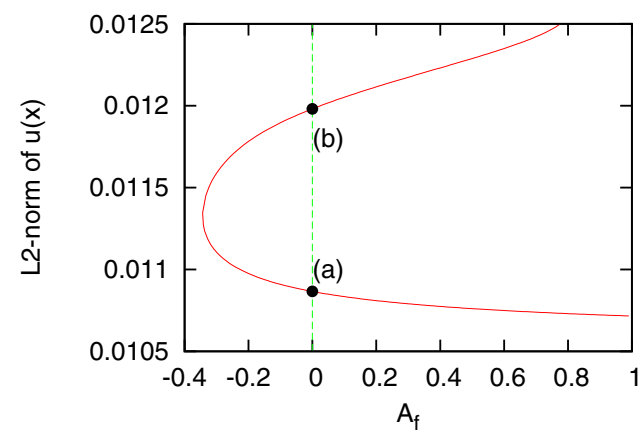

FIG. 2. (Color online) The trajectory of continuation projected onto a $A_{f}-\|u\|$ space. Each of two labeled solutions (a) and (b) on the line $A_{f}=0$ denotes a solution to SHE. The continuation is continued after the filter amplitude $A_{f}$ becomes zero and yields a solution labeled (b). The profiles of these solutions are shown in Fig. 3.

difficult to find. Indeed, we find an isolated and closed solution branch. Since isolated solution branches cannot be found by the weakly nonlinear framework, this success indicates an advantage of our method.

\section{A. Homoclinic snaking branches}

In this subsection we apply our method to SHE in order to obtain a localized steady solution belonging to the snaking branches. Although the branches contain stable localized solutions for a parameter region, the attracting basins of them are very small, and thus it is almost impossible to obtain these localized solutions by DNSs with arbitrary initial conditions.

Before adapting our method, the setting of system is described. We consider SHE in a region $[0, L], L=180$, and impose the fixed boundary conditions $u(0)=u(L)=0$. The parameter $r$ is set to -0.669 in this subsection, and -0.633 is used in the next subsection. Time evolutions are solved by use of the quasispectral method with the classical fourth-order Runge-Kutta method. We regard a steady point of DNS as a steady solution to the equation, so the DNS code is used also in continuation processes.

We describe the practical details of our method hereafter. The first step of our method is to introduce the damping term. The unfiltered region $\Omega$ is set to be $[80,100]$, and the filter function $H(x)$ is smoothed with $\sigma^{2}=0.01$. The amplitude of the filter $A_{f}$ is set to be 1 .

The second step is to obtain a solution to the filtered equation (1) with $F[u]$ of Eq. (4). This equation has a stable localized steady solution with these parameters. The initial condition of this DNS is the stable, steady sinelike solution of nonfiltered equation (4), which is not spatially localized but spatially extended. Such a localized solution to the filtered equation exists while $r \lesssim-0.72$. This lower limit almost agrees with that of the snaking branches. Since the spatial period of the sinelike solution is $2 \pi$, these localized solutions to the filtered equation contain almost three periodic components.

The third step is a continuation process. The parameter traced in this continuation is the filter amplitude $A_{f}$, and the parameter $r$ is fixed. The result of the continuation is shown in Fig. 2, which displays the trajectory of the continuation

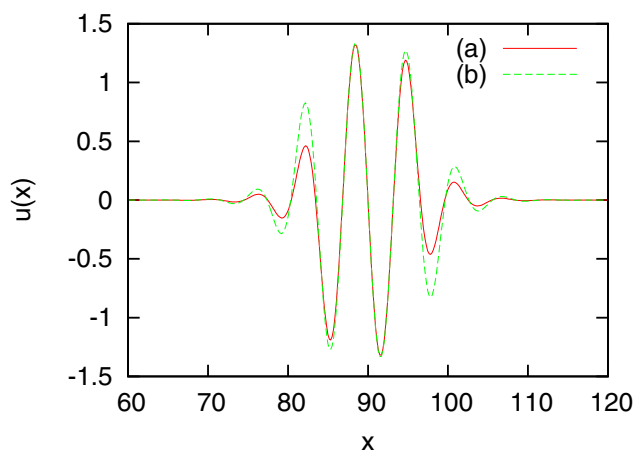

FIG. 3. (Color online) The profiles of the solutions obtained in the continuation shown in Fig. 2. Since the tails of the solutions decay exponentially, these solutions are almost localized in [70,110] and have an exponentially decaying tail.

projected onto a $A_{f}-\|u\|$ plane. Here $\|\cdot\|$ denotes the $\mathrm{L}^{2}$ norm. The trajectory crosses the line $A_{f}=0$ twice. Although a solution to SHE is obtained when the trajectory crosses the line first and thus the damping filter method finishes at this time, we find that the trajectory turns back and crosses the line $A_{f}=0$ again. Eventually, we successfully obtain two solutions to SHE, and the profiles of them are shown in Fig. 3. These solutions are localized almost in [70,110], which is larger than $\Omega=[80,100]$. This fact indicates that $\Omega$ is only a guide for obtaining a spatially localized solution, which obeys not the damping filter but the original equation.

\section{B. An isolated closed branch}

We execute the same procedure for various values of the parameter $r$. For most of $r$ it yields continuation trajectories and solutions to SHE similar to those shown in the previous section. However, we also find quite different behaviors in some cases, one of which we focus on in this subsection.

As an initial guess we use a solution to the filtered equation obtained by a continuation with the parameter $r$ started from the solution to the filtered equation used in the previous section. The other parameters are the same as those of the previous section: $\Omega=[80,100]$ and $A_{f}=1$.

The result of the continuation with $A_{f}$ starting from this starting solution is displayed in Fig. 4. Compared with Fig. 2, the continuation trajectory is very complicated, especially in the region $A_{f}<0$. The two solutions on the line $A_{f}=0$ are labeled as (c) and (d), and their profiles are displayed in Fig. 5.

A difference between the solutions (c) and (d) can be observed in its profile: The solution (c) is a single-pulse solution like the solutions (a) and (b). On the other hand, the solution (d) seems to be a combination of two antisymmetric pulse solutions. There exists the definitive difference in their solution branches as shown in Fig. 6. This figure shows that the solutions (a), (b), and (c) belong to the snaking branches, but the solution (d) belongs to an isolated closed branch. Such closed isolated solution branches are called "isolas" in Ref. [17].

It should be noted that two distinct branches are connected by the continuation with $A_{f}$. Moreover, they connect through 


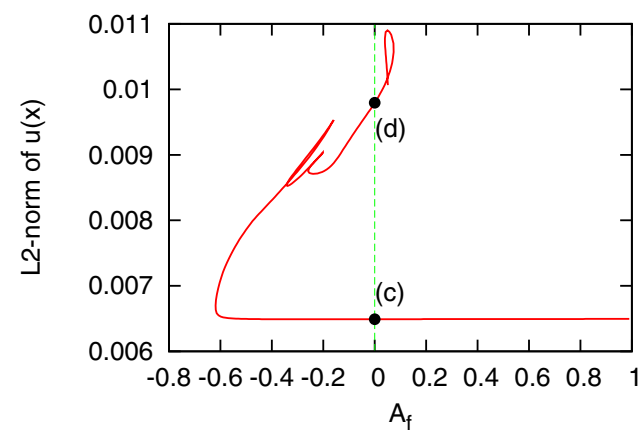

FIG. 4. (Color online) The trajectory of continuation projected onto $A_{f}-\|u\|$ starting from an unstable solution to the filtered equation. This continuation also cross the line $A_{f}=0$ twice, but a complicated path is realized. Two labeled points (c) and (d) on the line $A_{f}=0$ are also solutions to SHE, and their profiles are shown in Fig. 5. As noted in the text, a switching between solution branches occurrs in this complicated part of the trajectory.

the region where $A_{f}$ becomes negative, where the term $-A_{f} H(x) u$ works as an excitation term. Thus the connection can be regarded as a result of the instability caused by this term. Since $u(x)$ has an exponentially decaying tail, this instability occurs only around the edge of $\Omega$. So this linear exitation is also spatially selective.

\section{STREAMWISE-LOCALIZED SOLUTIONS}

In this section a streamwise-localized solution, in other words, a solution localized in its moving direction, is studied with the Kuramoto-Sivashinsky equation (KSE) as follows:

$$
\frac{\partial u}{\partial t}=F[u]=-u \frac{\partial u}{\partial x}-\frac{\partial^{2} u}{\partial x^{2}}-\frac{\partial^{4} u}{\partial x^{4}} .
$$

It should be noted that KSE has no localized equilibrium solution whose tail decays exponentially. If we adapt our method to Eq. (5), the continuation about $A_{f}$ yields the flat solution $u=0$ before $A_{f}$ reaches zero. So we seek a streamwise-localized traveling-wave solution (TWS) such that $u(x, t)=\hat{u}_{0}(x-c t)$, satisfying boundary conditions $\hat{u}_{0}(x-$ $c t \rightarrow \pm \infty) \rightarrow 0$.

In contrast to the case of spanwise-localized solutions, there are two issues in this case: One is a treatment of the propagation

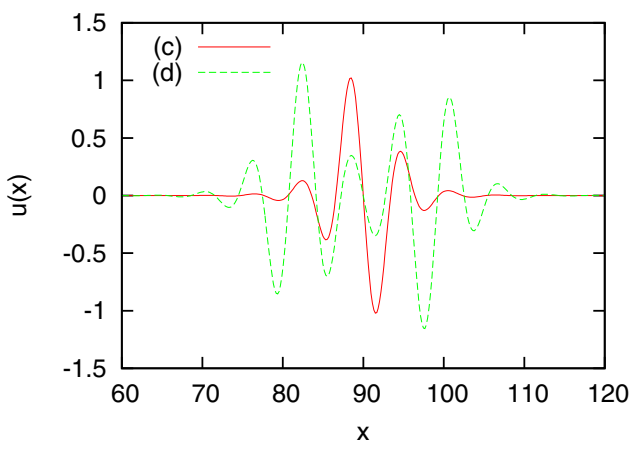

FIG. 5. (Color online) The profiles of the solutions (c) and (d). In contrast to Fig. 3, the profiles of the solutions (c) and (d) qualitatively differ.

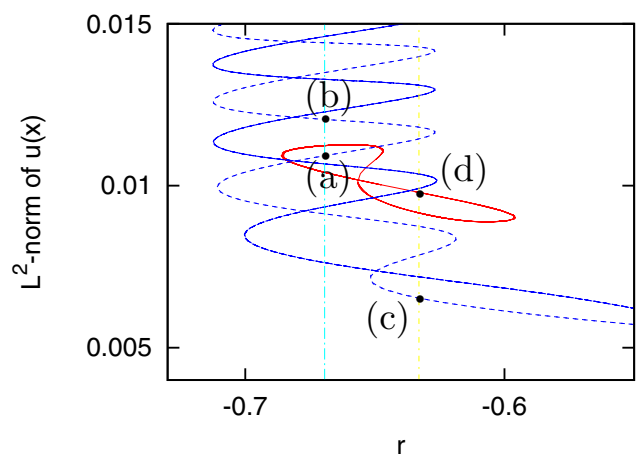

FIG. 6. (Color online) The snaking branches and isolas projected onto a $r-\|u\|$ plane. The snaking branches consist of two coupled branches, and solutions (a), (b), and (c) belong to the same (dashed blue line). The red (dark gray) figure-eight-shaped line denotes the isolas containing the solution (d). Such eight-figured branches can be seen also in the two-pulse solutions to SHE [17]. This similarity tells that the solution (d) should be regarded as a connected two-pulse solution.

velocity of the solution, and the other is a breakdown of the localization. These issues will arise in more general cases since they are based on Galilean and translational invariances. In the following subsections a solution to these issues is described.

\section{A. A treatment of the propagation velocity}

Since a TWS travels downstream, its localized region must accompany. In order to obtain a localized TWS by our method, it is necessary to introduce a moving damping filter or a steady damping filter in a moving frame, and we chose the latter. Then the equation becomes as follows:

$$
\frac{\partial \hat{u}}{\partial t^{\prime}}=-(\hat{u}-c) \frac{\partial \hat{u}}{\partial x^{\prime}}-\frac{\partial^{2} \hat{u}}{\partial x^{\prime 2}}-\frac{\partial^{4} \hat{u}}{\partial x^{\prime 4}}-A_{f} H\left(x^{\prime}\right) \hat{u},
$$

where $x^{\prime}=x-c t, t^{\prime}=t$, and $\hat{u}\left(x^{\prime}, t^{\prime}\right)=u(x, t)$. We seek a steady solution satisfying $\partial_{t^{\prime}} \hat{u}=0$ in this frame. However, the velocity of the moving frame $c$ is unknown unless the solution is obtained. Since KSE does not allow their solutions to continuously depend on $c$, a specific value $c_{0}$ with which a solution $\hat{u}_{0}\left(x-c_{0} t\right)$ exists must be determined simultaneously.

Such a situation sometimes occurs when obtaining a TWS to an equation $\partial_{t} u=F[u]$. In these cases, this problem is usually resolved by regarding $c$ as an unknown variable and solving $-c \partial_{x} u=F[u]$ for $u(x)$ and $c$. Since the translational invariance reduces the degree of freedom, this simultaneous equation can be solved. In our method, however, the damping filter breaks Galilean and translational invariances, thus the usual technique cannot be adapted.

Although TWSs to KSE do not continuously depend on $c$, our results show that TWSs to the filtered equation do. This fact reflects the breaking of the translational invariance by the filter. The details are discussed in the last part of this section. Thus the propagation velocity $c$ becomes one of the control parameters of the solution and can be chosen arbitrarily in a certain range. See also the schematic view in Fig. 7.

Then the other issue arises: How do we obtain the specific value $c_{0}$ ? Although there is a range of $c$ in which the solution 


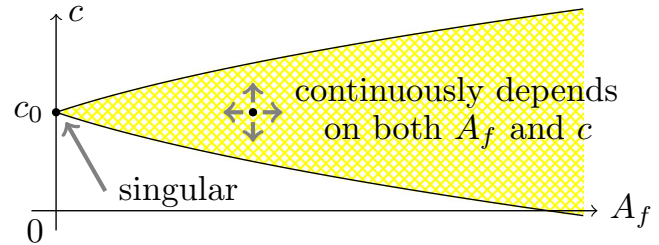

FIG. 7. (Color online) An image for the continuous existence of solutions and singularity at $A_{f}=0$. The existence of the solution is displayed in the $A_{f}-c$ plane. The solution exists continuously on the yellow (thin gray) cross-hatched region and cannot be traced beyond its rim. There is no guarantee that this solution branch connects to the line $A_{f}=0$. If it does, only a point is allowed because the solution cannot exist continuously on the line $A_{f}=0$.

exists continuously, this range becomes narrower and narrower as $A_{f}$ goes to zero and, finally, converges to a point on $A_{f}=0$. We get over this issue by imposing an implicit relationship between $c$ and $A_{f}$. This technique to impose the restriction is a key point to obtain a streamwise-localized TWS. The details are discussed in the next subsection with our data.

\section{B. Adapting the damping filtering method to the KSE}

In the first step of the damping filtering method, a localized region $\Omega$ is determined. We set the system size to $L=200$ and the localized region $\Omega=[97,103]$ in order to obtain a one-peak TWS. The filter function $H(x)$ is smoothed by a Gaussian with $\sigma^{2}=0.01$ and the filter amplitude $A_{f}$ is set to 4.8 .

In the second step, a solution to the filtered Eq. (1) is obtained to start the continuation. As notated in the previous subsection, we can choose an arbitrary propagation velocity $c$ in a certain range, and we choose $c=0$ here. We execute a DNS of nonfiltered equation (6) to produce a spatiotemporal chaotic field, which is used for an initial condition of a DNS of Eq. (6). This yields a stable solution, which is labeled as (a). These DNSs are solved by use of the quasispectral method with the classical fourth-order Runge-Kutta method. Although a localized solution has an exponentially decaying tail and does not get exactly to zero in a finite distance, we regard small values comparable to the truncation error as zero and assume that the fixed boundary conditions $u(0)=u(L)=0$ are satisfied. We use a sine transform to ensure this condition with $N=2048$ modes.

The third step is a continuation process (Fig. 8). Since the propagation velocity $c$ becomes a continuous parameter of the solution on $A_{f}>0$ region, this continuation becomes essentially two dimensional. It is almost impossible to obtain a full two-dimensional solution branch because of the numerical cost; therefore, we introduce a path on the $A_{f}-c$ space as follows.

As noted above, if $c$ is fixed to zero, then a continuation with $A_{f}$ yields the flat solution $u=0$ before $A_{f}$ reaches zero. In order to avoid this dead end, we first fix $A_{f}$ to 4.8 and execute a continuation with $c$. The solution is traced up to $c \simeq 1.21$, which is labeled as (b). This value of $c$ is determined by trial and error here, but a more practical criterion will be discussed in a future work.

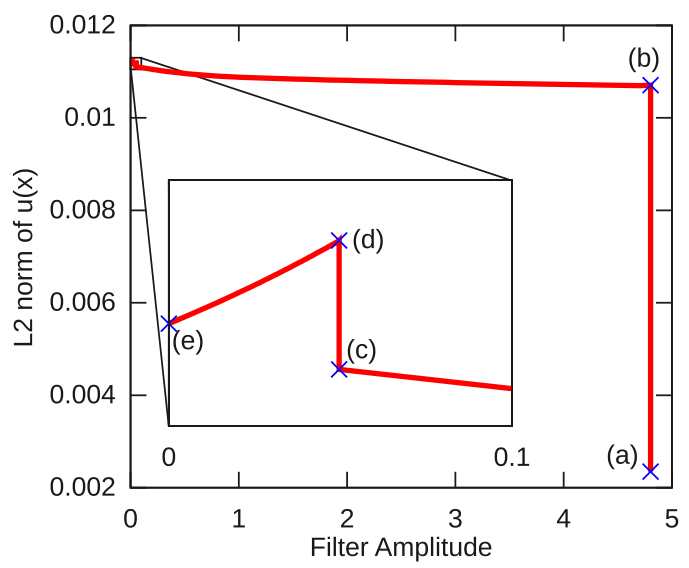

FIG. 8. (Color online) The trajectory of the continuation projected onto the $A_{f}-\|u\|$ plane. A closeup around $A_{f}=0$ is also shown.

The following continuation procedure is delicate because the uniqueness of the propagation velocity $c$ must recover when $A_{f}=0$. In short, the procedure consists of three steps (see Fig. 9) as follows: (i) $A_{f}$ is reduced around 0.05 while $c$ is fixed. (ii) $A_{f}$ is fixed and $c$ is adjusted to an "appropriate" value $c=c\left(A_{f}\right)$. (iii) $c$ and $A_{f}$ are traced simultaneously keeping the "appropriate" condition $c=c\left(A_{f}\right)$.

In the step (i), we fix $c$ to 1.21 and execute a continuation with $A_{f}$. This continuation leads $A_{f}$ around 0.01 , but $A_{f}$ cannot reach 0 . In this continuation the profile of the solution changes as shown in Fig. 10. We define a characteristic length of the tail of the solution as the inverse of its decaying rate. As $A_{f}$ decreases, it becomes longer and an oscillation starts to appear around the left boundary. If this tracing is continued further, the oscillation increases and the solution may not be kept localized. Such nonlocalized solutions also cannot be traced until $A_{f}=0$. This shows that a localized TWS to KSE cannot be obtained using only the continuation with $A_{f}$.

In order to avoid the oscillation around the boundary, we focus on the tail of the solution. In Fig. 10, the oscillation seems

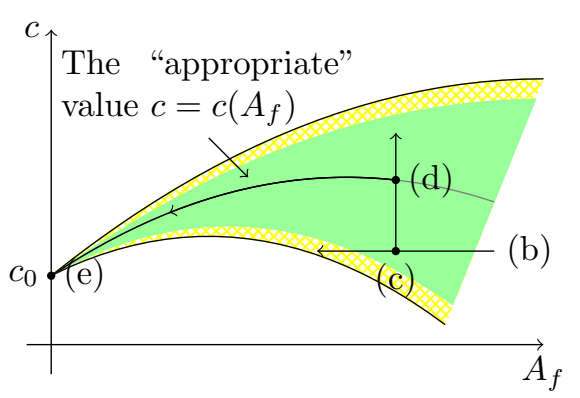

FIG. 9. (Color online) A schematic view of the parameter dependency of the solution around $A_{f}=0$. The yellow (thin gray) cross-hatched region denotes one where a solution continuously exists, and the green (thick gray) region denotes one where a "localized" solution continuously exists. Here a "localized" solution means that it does not have the oscillation around the boundary. The three steps are as follows: (i) (b) $\rightarrow$ (c), (ii) (c) $\rightarrow$ (d), and (iii) $(\mathrm{d}) \rightarrow(\mathrm{e})$. 


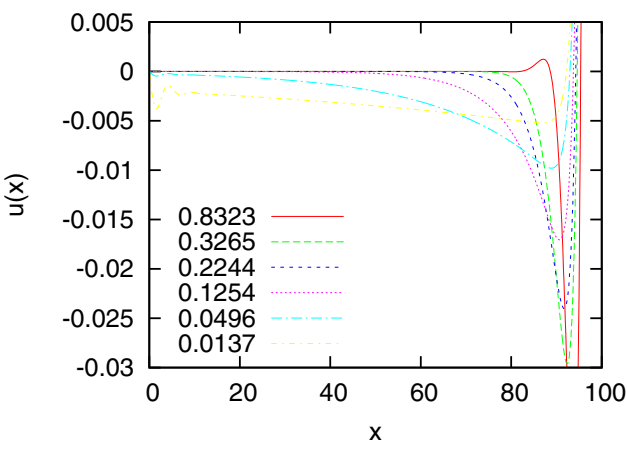

FIG. 10. (Color online) The left tails of the solutions obtained while the tracing (b) to (c) whose $A_{f}$ is $0.8323,0.3265,0.2244$, $0.1254,0.0496$, and 0.0137 , respectively. As $A_{f}$ decreases, the characteristic length of the tail becomes longer and longer. Finally, an oscillation appears around the left boundary due to the boundary condition.

to appear when the tail loses its flat part, for example, at $A_{f}=$ 0.2244 where its flat part is around $[0,60]$ and $A_{f}=0.1254$ where it is around $[0,40]$ the oscillation does not appear. We conclude that the disappearance of the flat part, which may occur when $A_{f}<1.0$, is a precursor of the oscillation at the boundary. We call this decrease of the flat part "the weakening of the localization" and will discuss this mechanism in the next section.

We found that there exists a specific value $c$ for each $A_{f}$ such that the flat part recovers. Figure 11 shows the change in the tail of the solution while the continuation with $c\left(A_{f}\right.$ is fixed to 0.0496). At $c=1.2319$ the flat part recovers to be $[0,80]$. This continuation is step (ii), and the "appropriate" value is $c=1.2319$.

This "appropriate" value of $c$ varies with $A_{f}$. In other words, the "appropriate" relation $c=c\left(A_{f}\right)$ defines a path on the two-dimensional parameter space $c-A_{f}$. Along this path the solution has always a flat part. In order to trace the solution along the path $c=c\left(A_{f}\right)$, however, it is necessary to express the condition $c=c\left(A_{f}\right)$ numerically. It can be easily done

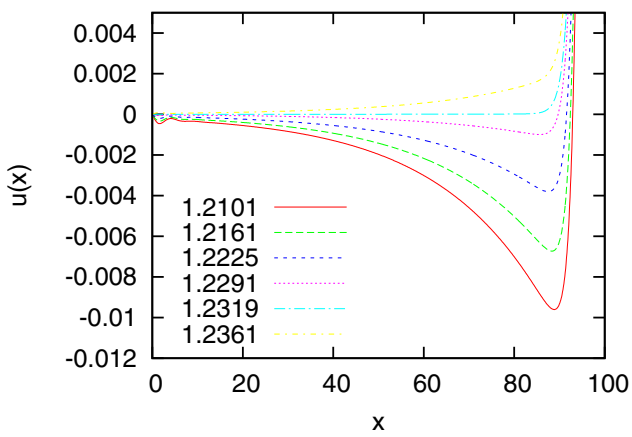

FIG. 11. (Color online) A part of the profile of solutions obtained while the tracing (c) $\rightarrow$ (d) whose traveling velocity $c$ is 1.2101(c), 1.2161,1.2225,1.2291,1.2319(d),1.2361. The mechanism of this weakening of the localization is presented in Sec. IV C.

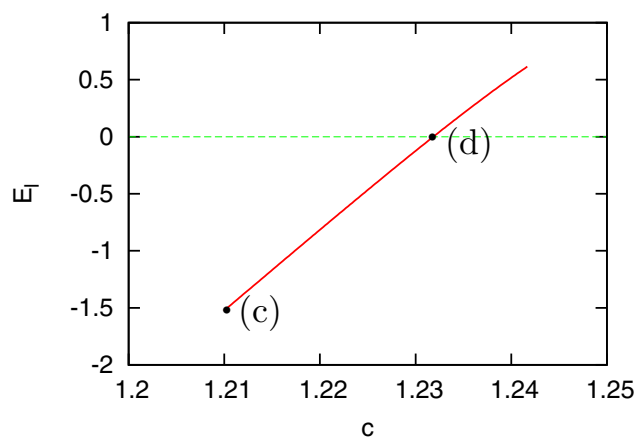

FIG. 12. (Color online) $E_{l}$ is plotted against the traveling velocity $c . E_{l}$ measures how long the tail of the solution is. $E_{l}(c)$ cross the line $E_{l}=0$, and this point is labeled (d). This crossing behavior is also argued in Sec. IV C.

with the following integral value:

$$
E_{l}=\int_{0}^{l} u(x) d x,
$$

where $l$ is chosen to be in the tail part. The change of $E_{l}$ in the continuation mentioned above is shown in Fig. 12. The relation $c=c\left(A_{f}\right)$ is now implicitly defined by $E_{l}\left(c, A_{f}\right)=$ 0 . Then we can continuate the branch $u\left(x ; c\left(A_{f}\right), A_{f}\right)$ with $A_{f}$. This conditional continuation can be implemented by ue of a $(N+1)$-dimensional arc-length method, and we have succeeded in tracing the solution to $A_{f}=0$ within a numerical accuracy. This solution is labeled as (e) in Fig. 8, and its profile is displayed in Fig. 13. Its propagation velocity $c_{0}=c(0)$ is equal to 1.2143. This is the same solitary-wave solution as that shown in Fig. 4(c) of Ref. [18].

\section{Why the tail of the solution becomes longer?}

In this subsection the mechanism of the weakening of the localization is considered. This weakening behavior enable us to obtain an implicitly defined path in $A_{f}-c$ space. In order to generalize this technique to more complicated systems such as channel flows or pipe flows, it is necessary to investigate its details more precisely.

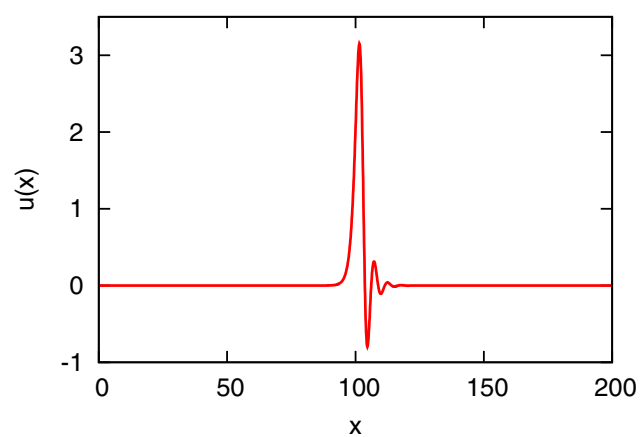

FIG. 13. (Color online) The profiles of TWS to Eq. (5). It takes a nonzero value on a region $[90: 115]$, which is much wider than $\Omega=[97: 103]$. 
A steady localized solution $u(x)$ to Eq. (6) satisfies the ordinary differential equation

$$
\frac{d^{4} u}{d x^{4}}+\frac{d^{2} u}{d x^{2}}+(u-c) \frac{d u}{d x}+A_{f} H(x) u=0 .
$$

Here hats and primes are omitted for convenience. Regarding $x$ as a virtual time, this equation defines a four-dimensional dynamical system. Then a localized solution $u(x)$ to (6) corresponds to a homoclinic orbit connecting the saddle point of this dynamical system, and the tails of the solution describe the asymptotic behavior of the homoclinic orbit from and to the saddle point. It should be noted that this reinterpretation has less compatibility with the fixed boundary condition $u(0)=u(L)=0$ since we now consider the asymptotic behaviors in an infinite region $(-\infty, \infty)$ instead of the bounded region $[0, L]$. However, the following arguments are all valid whenever $u(x)$ decays fast enough to be negligible comparing with the truncation error.

This dynamical system has a trivial fixed point (saddle point) $\left(u, \partial_{x} u, \partial_{x x} u, \partial_{x x x} u\right)=(0,0,0,0)$ corresponding to the solution $u(x)=0$ to Eq. (6). The localized solution $u(x)$ can be regarded as a homoclinic trajectory of this trivial fixed point. Then the tail of the localized solution can be analyzed by the eigenvalues of Jacobi matrix of this dynamical system at the fixed point. Since $H(x)$ is equal to 1 in the tail region, the eigenequation of the Jacobi matrix becomes the following:

$$
\lambda^{4}+\lambda^{2}-c \lambda+A_{f}=0 .
$$

This quartic equation has two real roots $\lambda_{0}, \lambda_{1}$ and two complex roots $\lambda_{ \pm}$. Since we focus on the case $A_{f} \ll 1$, we first consider the case $A_{f}=0$, and then a perturbation expansion about $A_{f}$.

When $A_{f}=0$, the eigenvalues are $\lambda_{0}=0$ and three roots of a cubic equation $\lambda^{3}+\lambda-c=0$. This cubic equation has a real root $\lambda_{1}$ and two complex roots $\lambda_{ \pm}=\left(-\lambda_{1} \pm i \sqrt{3 \lambda_{1}^{2}+4}\right) / 2$. The real nonzero root $\lambda_{1}$ is positive when $c>0$ and negative when $c<0$, and here we consider the $c>0$ case. Then the left and right tails of the solution can be written as follows:

$$
\begin{gathered}
u_{L}(x)=A_{1} e^{\lambda_{1} x}, \\
u_{R}(x)=A_{+} e^{\lambda_{+} x}+A_{-} e^{\lambda_{-} x} .
\end{gathered}
$$

The coefficients $A_{1}, A_{+}, A_{-}$are determined in the nonlinear region. Since $\exp \left(\lambda_{0} x\right)$ does not goes to zero as $x \rightarrow \pm \infty$, $u(x)$ cannot contain this term.

When $0<A_{f} \ll 1$ the zero eigenvalue is modified as $\lambda_{0}=$ $A_{f} / c+O\left(A_{f}^{2}\right)$. Then the left tail of the solution can be written as follows:

$$
u_{L}(x)=A_{0} e^{\lambda_{0} x}+A_{1} e^{\lambda_{1} x} .
$$

The coefficients $A_{0}$ and $A_{1}$ are also determined in the nonlinear region and depend both on $c$ and $A_{f}$. Each of the terms in Eq. (12) defines a tail whose characteristic length is $1 / \lambda_{0}$ and $1 / \lambda_{1}$, and the realized tail is a superposition of them. As $A_{f}$ goes to zero, the characteristic length $1 / \lambda_{0}=c / A_{f}$ diverges. Thus the modified eigenvalue $\lambda_{0}$ is the origin of the weakening, i.e., the long tail.

Next, we consider why we can obtain a solution with a short tail by the condition $E_{l}=0$ for every small $A_{f}$. Using
Eq. (12), $E_{l}$ can be written as follows:

$$
E_{l} \simeq \int_{-\infty}^{l} u_{L}(x) d x=\frac{A_{0}}{\lambda_{0}} e^{\lambda_{0} l}+\frac{A_{1}}{\lambda_{1}} e^{\lambda_{1} l} .
$$

Since $1 / \lambda_{0} \gg 1 / \lambda_{1}$ when $A_{f} \ll 1$, the first term in Eq. (12) is dominant except for a region near the nonlinear region. Thus the first term in Eq. (13) is rather dominant for an appropriate $l$. Then $E_{l}$ roughly measures $A_{0}$, and $E_{l}=0$ is realized when $A_{0}$ is zero where the tail by $\lambda_{0}$ disappears. A more precise argument is also possible. The condition $E_{l}=0$ can yield the condition of $A_{0}$ as follows:

$$
\begin{aligned}
A_{0} & =-\frac{\lambda_{0}}{\lambda_{1}} A_{1} e^{\left(\lambda_{1}-\lambda_{0}\right) l} \\
& =-\frac{A_{f}}{c \lambda_{1}} A_{1} e^{\left(\lambda_{1}-\lambda_{0}\right) l}+O\left(A_{f}^{2}\right) .
\end{aligned}
$$

Thus $A_{0}$ is not exactly zero while $A_{f}>0$. However, since $\lambda_{0}$ goes to zero as $A_{f} \rightarrow 0, A_{0}$ satisfying this condition also goes to zero.

The essence of the above arguments is the existence of the zero eigenvalue $\lambda_{0}$ and its modification due to the damping. The modified zero eigenvalue introduces another degree of freedom in the determination of tails of solutions. Calculating the eigenvector of the zero eigenvalue, it corresponds to a uniform level raising of the velocity field, $u(x) \mapsto u(x)+\delta c$, so it corresponds to Galilean invariance. In other words, the reason this small eigenvalue appears is the breakdown of Galilean invariance. This fact indicates that such a weakening of the localization is expected to occur whenever a streamwisellocalized TWS is going to be obtained in Galilean-invariant systems by use of our method.

At last, we conclude this section with an error estimate. Since the continuous parameter dependence on $c$ disappears when $A_{f}=0$, the continuation becomes unstable as $A_{f}$ goes to zero. Although the point $A_{f}=0$ is a singular point in this continuation problem, $A_{f}$ can get an arbitrary value as small as the numerical accuracy allows. Indeed, we get $A_{f} \sim 10^{-10}$ in the conditional continuation. This is as small as the threshold for the Newton method, $\varepsilon_{\text {Newton }}$. Then the continuated solution can be regarded as a solution to KSE within the numerical error $\varepsilon_{\text {Newton }}+A_{f}\|u\|$.

\section{CONCLUDING REMARKS}

In this paper we introduce the damping filter method for obtaining spatially localized solutions. We adapt our method into two fundamental cases. First, in Sec. III, we considered localized solutions to the SHE. Then our method can not only reproduce known solutions but also obtain another spatially localized solution which belongs to a closed isolated solution branch. Next, in Sec. IV, we consider a streamwise-localized TWS to the KSE. In this case, since the propagation velocity $c$ is unknown, we have to continuate the solution with $c$ and the filter amplitude $A_{f}$. In order to make continuation onedimensional we introduce an implicit condition about the tail of solutions. Here we reinterpret these result from a general point of view in order to adapt our method into more general cases. 
The most interesting result in the Sec. III is the connection between two distinct solution branches. We show that two solution branches are connected with each other through the continuation with the filter amplitude $A_{f}$. We first introduce the filter term $-A_{f} H(x) u$ in order to obtain a guess at spatially localized solutions. However, it works as an excitation when $A_{f}<0$. This excitation only works near the localized region where both $u(x)$ and $H(x)$ are nonzero. This causes an instability which may lead to another localized solution. Indeed, we obtain the solution (d) in Sec. III only by the continuation with $A_{f}$. This spatially selective excitation mechanism has an advantage in searching spatially localized solutions. The result for SHE indicates that if another localized solution exists near the localized solution already obtained in the phase space, they may connect through this excitation mechanism. So our method may enable us to search localized solutions automatically.

In Sec. IV, we dealt with a spatially localized TWS to KSE. The main issue of this section is a treatment of the invariances. The damping filter term breaks the translational and Galilean invariances, but they recover when the filter disappears. This singular behavior is avoided by imposing the condition $c=$ $c\left(A_{f}\right)$ by use of the implicit condition $E_{l}\left(c, A_{f}\right)=0$.

This artificial condition can be reinterpreted as a critical line of the orbit-flip bifurcation [19]. For fixed $A_{f}$ an orbit-flip bifurcation occurs when $A_{0}$, defined in Sec. IV C, changes its sign with increasing $c$, see Figs. 9 and 11. Moreover, if $A_{f} \ll$ 1, the relation $A_{0} \sim A_{f} \sim 0$ holds in $c=c\left(A_{f}\right)$ because of
Eq. (14). Therefore we can infer that the condition $c=c\left(A_{f}\right)$ corresponds to the critical line of the orbit-flip bifurcation in the four-dimensional ODE system [Eq. (8)].

To implement the critical condition directly, we can utilize an algorithm for tracking the orbit-flip bifurcation in AUTO [20]. This algorithm replaces the condition $E_{l}=0$ with an orthogonal condition to keep the tangency between the homoclinic orbit and the leading eigenspace. Moreover, this method clarifies the mathematical meaning of our condition. However, since it is designed for a homoclinic orbit to ODE, it might be difficult to apply it for spatially two- or threedimensional PDE systems. We thus expect that our method using $E_{l}$ will be more suitable for the dynamical systems approach to turbulence.

\section{ACKNOWLEDGMENTS}

We thank T. Ogawa for invaluable comments regarding the Swift-Hohenberg equation. We also appreciate the referee referring us to the orbit-flip bifurcation. This work was supported by the Grants for Excellent Graduate Schools "The Next Generation of Physics, Spun from Universality and Emergence" from the Ministry of Education, Culture, Sports, Science, and Technology (MEXT) of Japan and also partially by JSPS KAKENHI Grant No. 22540386. Part of the numerical calculations were carried out on SR16000 at YITP at Kyoto University.
[1] G. Kawahara, M. Uhlmann, and L. van Veen, Annu. Rev. Fluid Mech. 44, 203 (2012).

[2] J. Jiménez and P. Moin, J. Fluid Mech. 225, 213 (1991).

[3] T. Itano and S. Toh, J. Phys. Soc. Jpn. 70, 703 (2001).

[4] J. D. Skufca, J. A. Yorke, and B. Eckhardt, Phys. Rev. Lett. 96, 174101 (2006).

[5] G. Kawahara and S. Kida, J. Fluid Mech. 449, 291 (2001).

[6] T. M. Schneider, B. Eckhardt, and J. A. Yorke, Phys. Rev. Lett. 99, 034502 (2007).

[7] Y. Duguet, P. Schlatter, and D. S. Henningson, Phys. Fluids 21, 111701 (2009).

[8] T. M. Schneider, D. Marinc, and B. Eckhardt, J. Fluid Mech. 646, 441 (2010).

[9] M. Avila, F. Mellibovsky, N. Roland, and B. Hof, Phys. Rev. Lett. 110, 224502 (2013).

[10] J. Burke and E. Knobloch, Chaos 17, 037102 (2007).
[11] E. Knobloch, Nonlinearity 21, T45 (2008)

[12] M. Beck, J. Knobloch, D. J. B. Lloyd, B. Sandstede, and T. Wagenknecht, SIAM J. Math. Anal. 41, 936 (2009).

[13] T. M. Schneider, J. F. Gibson, and J. Burke, Phys. Rev. Lett. 104, 104501 (2010).

[14] A. Bergeon and E. Knobloch, Phys. Fluids 20, 034102 (2008).

[15] M. Nagata, J. Fluid Mech. 217, 519 (1990).

[16] J. Jiménez and M. P. Simens, J. Fluid Mech. 435, 81 (2001).

[17] J. Knobloch, D. J. B. Lloyd, B. Sandstede, and T. Wagenknecht, J. Dyn. Differ. Eq. 23, 93 (2011).

[18] D. Michelson, Physica D 19, 89 (1986).

[19] A. R. Champneys, Y. A. Kuznetsov, and B. Sandstede, Int. J. Bifurcation Chaos 06, 867 (1996).

[20] E. J. Doedel, R. C. Paffenroth, A. R. Champneys, T. F. Fairgrieve, Y. A. Kuznetsov, B. E. Oldeman, B. Sandstede, and X. J. Wang [http://indy.cs.concordia.ca/auto/]. 